\title{
Reproductive safety assessment of Thymus vulgaris L. extract and quantification of thymol sulfate in pregnant rats and fetuses using a validated LC/MS method of analysis
}

\author{
Zaineh Q. Tafesh ${ }^{1}$, Kenza A. Mansoor ${ }^{1}$, Nidal A. Qinna ${ }^{2}$, Feras D. El-Hajji ${ }^{3}$, Tawfiq A. Arafat ${ }^{4}$, Luay F. Abu-Qatouseh ${ }^{1}$, \\ Bayan Y. Ghanim², Iman A. Basheti ${ }^{3}$, Eyad M. Mallah ${ }^{1^{*}}$ \\ ${ }^{1}$ Faculty of Pharmacy and Medical Sciences, University of Petra, Amman, Jordan. \\ ${ }^{2}$ University of Petra Pharmaceutical Center (UPPC), University of Petra, Amman, Jordan. \\ ${ }^{3}$ Faculty of Pharmacy, Applied Science Private University, Amman, Jordan. \\ ${ }^{4}$ Jordan Center for Pharmaceutical Research (JCPR), Amman, Jordan.
}

\section{ARTICLE INFO \\ Received on: 09/05/2020 \\ Accepted on: 22/10/2020 \\ Available online: 05/01/2021}

Key words:

Thyme, essential oil, toxicity, fetus, pregnancy, cough syrups.

\begin{abstract}
The current study aimed to investigate the safety of ingesting standardized thyme extract (Thymus vulgaris L.) during pregnancy in rats. For this purpose, postmortem examination and thymol sulfate (TS) concentration in maternal and fetal effluent extracts were determined. TS concentrations were analyzed by developing and validating a new liquid chromatography-mass spectrophotometer method for quantification. Three dose levels of 1-, 10-, and 20-fold (x) of the recommended dose of standardized thyme extract in parallel to thymol were administered to evaluate the development of rat fetuses from the 5th to the 19th day of gestation. Blood samples from pregnant rats and fetal tissue samples were analyzed to detect the presence of TS that is a major thymol metabolite. The average concentration of TS detected in pregnant rats' plasma-administered thyme extract was $648 \pm 106 \mathrm{ng} / \mathrm{ml}$ at 1x dose compared to $3,429 \pm 865 \mathrm{ng} / \mathrm{ml}$ for its equivalent amount of thymol compound. Quantities of TS in the dams and fetal tissue were found to increase in a dose-dependent manner. The administration of the $1 \mathrm{x}$ and $10 \mathrm{x}$ doses of the extract resulted in a significant increase in the fetal and placental weights. However, 20x dose of both thyme extract and thymol compound exhibited an increase in the number of adsorbing sites in the uterus and reduced birth weights compared to control. In conclusion, TS was capable of crossing the placental barrier in rats and increased the resorption index when high and repeated doses of thyme extract were administered. The current investigation highlights the expected danger of exceeding the therapeutic dose when consuming thyme remedies during pregnancy.
\end{abstract}

\section{INTRODUCTION}

Most studies on herbal medicinal products are directed toward investigating their possible pharmacological effects and active constituents rather than focusing on their toxicity to the human body, especially during pregnancy. However, the use of most herbal remedies during pregnancy is cautioned, not only to pregnant women but to their fetuses that might also be at risk of toxicity following its consumption (Louik et al., 2010). Common

\section{"Corresponding Author}

Eyad M. Mallah, Faculty of Pharmacy and Medical Sciences, University of Petra, Amman, Jordan. E-mail: emallah@uop.edu.jo thyme or garden thyme is a herb of Thymus vulgaris L. or Thymus zygis L. that belongs to the Labiatae or also known Lamiaceae family (World Health Organization, 1999). Despite the diversity in thyme species, T. vulgaris $\mathrm{L}$. is the most commonly used in therapeutic dosage forms (Hosseinzadeh et al., 2015). Thymol (2-isopropyl-5-methylphenol), on the other hand, is a colorless crystalline substance that gives thyme its strong flavor and pleasant aromatic odor. Thyme herb extract (TE) and thyme oil are traditionally used to treat symptoms of bronchitis and whooping cough (Basch et al., 2004; Gavliakova et al., 2013).

Since thyme is usually consumed in considerable amounts as food, known as Za'atar in Arabic, it is described as "generally recognized as safe" (Basch et al., 2004). However, a study in 1980 showed that thymol had produced teratogenic 
effects in the developing chicken embryo (Verrett et al., 1980). Additionally, another study confirmed morphological toxic alterations in the histology of liver and thyroid tissues of mice exposed to $2 \mathrm{mg} / \mathrm{ml}$ of thymol (Leonardo et al., 2017). Data concerning thyme ingestion during pregnancy needs investigation on its effects in relevant endpoints of prenatal exposure on pregnant test animals and on the developing organism; this as recommended by the Organization for Economic Co-operation and Development guidelines on prenatal developmental toxicity study, which includes the assessment of maternal effects as well as death, structural abnormalities, or altered growth in the fetus (OECD/OCDE, 2018). In addition, determining the maternal/fetal effluent defined by the placental crossing of suspected chemical or metabolite is of major concern. Developing suitable and sensitive analytical methods to carry out quantitative analysis for active natural compounds and their metabolites is considered a key means toward assessing in vivo toxicokinetic and safety investigations in herbal medicine (Sharma and Sarkar, 2013; Woo et al., 2012). The inherent complexity and variability of herbal extracts have been a significant challenge for separation and detection methods during the analysis of herbal medicine (Marston and Hostettmann, 2009). Additionally, in pharmacokinetic and pharmacodynamic studies, qualification and quantification of metabolites of natural products in complex biological matrixes also require advanced analytical methods with high sensitivity and selectivity (Gray et al., 2010). In this context, tandem mass spectrometry has become a routine analytical technique in clinical practice for quantitative analysis of natural products (Wu et al., 2013). Prior to any liquid chromatography-mass spectrophotometer (LC/MS) analysis, extraction of the desired chemical constituents from the herbal mixtures or biological samples is required to get sample aliquots free of interferences and may also require concentrating the analyte for improved detection or better separation (Wu et al., 2013). Such analytical techniques should be developed and validated prior to implementation.

The current research aimed to evaluate the preclinical safety of thyme extract and its metabolite, namely thymol sulfate (TS), during pregnancy in both pregnant rats and their fetuses initially through observing its effects on the mothers and uterine content. In addition, quantification of TS was carried out utilizing a developed and valid LC/MS method in order to provide insight into the quantities of the metabolite that crossed the placenta reaching the fetus. Such an approach intends to reveal the concentrations that possibly alter the placental functions and therefore could affect the growth of fetuses. An objective of the study is to quantify thymol sulfate (a major thymol metabolite) in samples from mother and fetus through LC/MS.

\section{MATERIALS AND METHODS}

\section{Materials}

Thymi herba ethanolic extract (TE) (T. vulgaris L., 80\% native) containing $0.65 \%$ of thymol monoterpene was obtained from Martin Bauer Co. (Germany). Pure thymol (T) with more than $99.5 \%$ purity was obtained from Sigma Aldrich (United States). TS potassium salt was obtained from Toronto Research Chemicals (Canada), while the internal standards (IS), namely hydrochlorothiazide (HCT) along with 5\% formic acid (FA), ethyl acetate (EA), ammonium chloride, and methanol $(\mathrm{MeOH})$, were all purchased from Thermo Fisher (United States). All herbal materials were kept in dark amber containers at room temperature.

\section{In vivo dose preparation}

The first dose level of TE, i.e., the 1x dose, was calculated as $93.75 \mathrm{mg} / \mathrm{kg}$, which represents the human recommended daily dose (RDD) and prepared in distilled water (Basch et al., 2006). The RDD selection was based on prescribing up to $30 \mathrm{mg} /$ day of cough syrup $(15 \mathrm{~g} / 100 \mathrm{ml})$ to pregnant women by healthcare practitioners. On the other hand, since each $100 \mathrm{mg}$ of the standardized extract contains $0.65 \mathrm{mg}$ of thymol (T) according to the source of the extract supplier, the $1 \mathrm{x}$ dose of $\mathrm{T}$ was calculated as $0.60 \mathrm{mg} / \mathrm{kg}$ and prepared in a propylene glycol solution [polyethylene glycol (PG); BBC Chemicals, Torre Boldone, Italy]. All formulations were freshly prepared on the day of administration to ensure consistency and stability of the preparation.

\section{In vivo prenatal development parallel study design}

\section{Animal handling}

Adult male and female healthy Wister rats with an average age range of 10-12 weeks were housed in the Animal House of the Applied Science Private University (Amman, Jordan) in solid-bottom polycarbonate cages. The rats were accurately weighed and accommodated in an air-conditioned environment $\left(20^{\circ} \mathrm{C}-25^{\circ} \mathrm{C}\right)$, having a relative humidity of $30 \%-$ $70 \%$, and exposed to a 12-hours daily photoperiod cycle. Animals were offered a conventional laboratory diet and clean tap water $\mathrm{ad}$ libitum. Rats were examined daily for external signs of toxicity (diarrhea, weight loss, or bleeding) or behavioral abnormalities. All animal procedures and the study protocol were revised and approved by the ethical committee of the University of Petra, Amman, Jordan (ethical approval number: 2016/2017-66).

\section{Mating and pregnancy induction}

The polygamous design was adapted to induce pregnancy in rats where each female rat was caged with 2-3 males and monitored for any copulation behavior considering day 0 of gestation as the day on which formation of a vaginal plug was observed (OECD/OCDE, 2018). All pregnant rats were daily observed throughout the period of gestation for mortality, morbidity, general appearance, and abnormal behavioral changes.

\section{Study design}

Rats received daily oral doses of corresponding treatments using an oral gavage needle from the 5 th day of gestation until day $19^{\text {th }}$ day, which is defined as the period of structural development of the embryonic stage for rats (OECD/OCDE, 2018; Wang et al., 2014). The pregnant rats $(n=40)$ were marked and randomized into different groups, where rats in groups 1,2, and 3 were orally administered with 1,10 , and $20 \mathrm{x}$ doses of TE that are equivalent to $93.75,937.5$, and $1,875 \mathrm{mg} / \mathrm{kg}$, respectively, along with group 4 receiving distilled water $(5 \mathrm{ml} / \mathrm{kg})$ and serving as a placebo control for TE-treated groups. In addition, rats randomized in groups 5, 6, and 7 were administered with 1, 10, and 20x dose of $\mathrm{T}$ (equivalent to $0.6,6$, and $12 \mathrm{mg} / \mathrm{kg}$, resp.) along with group 8 receiving $P G$ solution as a placebo control for T-treated groups (Fig. 1). Rats 
were weighed weekly from gestation day 0 to the day of humane termination.

\section{Sample collection}

Caesarean procedure

On the 19th day, dams were sacrificed by cervical dislocation and blood samples were collected from rats by cardiac puncture and drawn into Ethylenediaminetetraacetic acid (EDTA) microtainer tubes. The tubes were directly centrifuged for 5 minutes at 4,000 rpm and plasma was separated into labeled tubes and stored at $-60^{\circ} \mathrm{C}$ until requested for analysis. Rats were then immediately prepared for a caesarean section and their uterus horns were removed and weighed. All placentas were isolated and grossly examined for changes in color or presence of lesions. Uteri were then carefully removed and accurately weighed. The number of live, dead, or resorbed fetuses was recorded and all fetuses were inspected for external malformations or teratogenic signs in the head, cleft palate, limbs, or tail (OECD/OCDE, 2018).

Fetuses and placentas were weighed for each litter. Thereafter, fetuses of each group were then pooled and homogenized using a polytron tissue homogenizer and then centrifuged for 5 minutes at 1,000 rpm. The supernatant of each pooled sample was then separated and properly labeled. Maternal and fetal extracted samples were preserved at $-60^{\circ} \mathrm{C}$ until LC/MS analysis of TS.

\section{Data presentation and analysis}

Relative uterus weight was calculated as organ to body ratio (using the terminal body weight) using the following equation:

Relative uterus weight $=$ absolute weight of uterus / weight of rat in the day of sacrefice

In addition, the following equation was used to calculate resorption index:

Resorption index $=$ total number of resorption sites / total number of implantation sites $\times 100$

All data points are represented as mean \pm standard error of the mean. The statistical significance of difference in mean of a normally distributed variable was assessed using analysis of variance, followed by Tukey's post-hoc test carried out using International Business Machines Corporation (IBM) statistical package for social science program version 25 (SPSS Inc., Chicago, IL). A value of $p<$ 0.05 was considered to be statistically significant.

\section{Chromatographic conditions and TS method analysis and validation}

Instrumentation and chromatographic condition

An high-performance liquid chromatography (HPLC) from Agilent Technologies, CA (model Agilent 1,200), was arranged, which comprised an online vacuum degasser, a solvent delivery system pump, an autosampler, and a thermostat column compartment combined with an MS detector from SCIEX Company (model API 4,000). The used column was ACE-C8 column $(50 \times 4.6 \mathrm{~mm}$ i.d. $5 \mu)$ obtained from Thermo Electron Corporation. The mobile phase was composed of $30 \% 0.5 \mathrm{mM}$ ammonium chloride: $70 \% \mathrm{MeOH}$ ratio, while the flow rate was adjusted to $0.65 \mathrm{ml} /$ minutes. The injection volume was $10 \mu \mathrm{l}$ and the temperature of the autosampler was adjusted to $25^{\circ} \mathrm{C}$ in addition to other chromatographic conditions summarized in Table 1.

Preparation of internal standard solution

A $1 \mathrm{mg} / \mathrm{ml}$ stock solution of the internal standard was prepared by dissolving $10 \mathrm{mg}$ of HCT in $10 \mathrm{ml}$ methanol and then further diluted to produce $2 \mu \mathrm{g} / \mathrm{ml} \mathrm{HCT}$ solution.

Preparation of the standard curve and QC samples of TS

The lower limit of quantification (LLOQ) was set at 40 $\mathrm{ng} / \mathrm{ml}$. Eight concentrations ranging from $40-3,000 \mathrm{ng} / \mathrm{ml}$ were prepared by spiking rat plasma samples with TS to construct its standard curve. A representative chromatogram and a standard curve are shown in Figure 2.

\section{Liquid-liquid extraction method}

Plasma samples $(0.2 \mathrm{ml})$ were transferred into Eppendorf tubes and spiked with $50 \mu \mathrm{l}$ of the IS solution and $75 \mu \mathrm{l}$ of $5 \%$ FA and vortexed for 1 minute. EA $(4 \mathrm{ml})$ was then added and the tubes were vortexed for 5 minutes and centrifuged for 4 minutes at $4,400 \mathrm{rpm}$. The samples were frozen for 30 minutes at $-40^{\circ} \mathrm{C}$ and then the supernatant organic layer was separated and evaporated

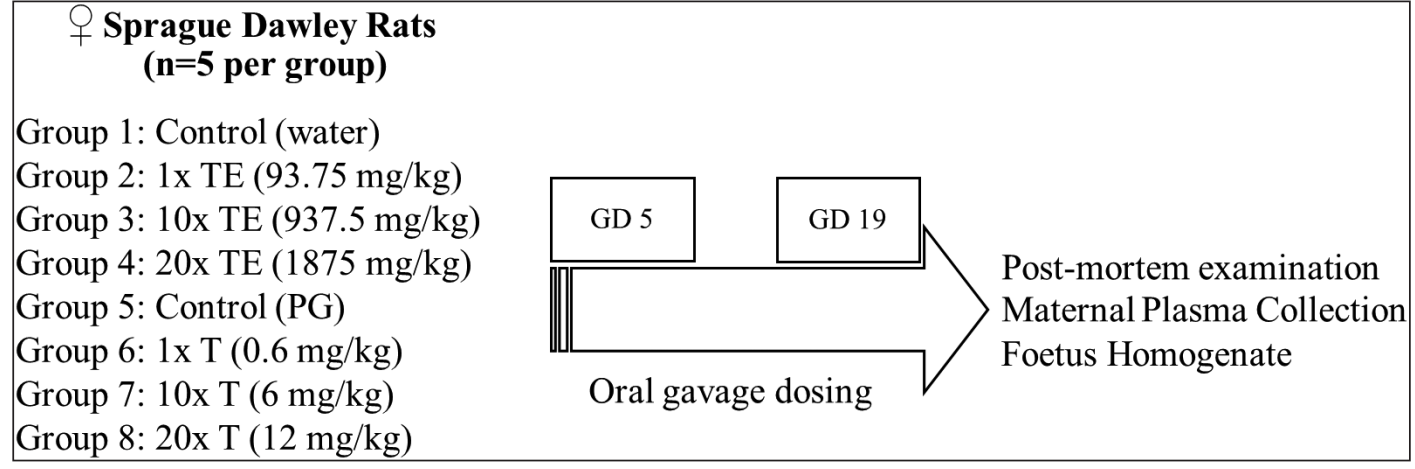

Figure 1. Schematic design of prenatal toxicity experiment on thyme extract (TE) and thymol $(\mathrm{T})$ in Sprague Dawley rats. PG $=$ polyethylene glycol; GD = gestation day. 
Table 1. LC/MS chromatographic conditions applied for the detection of TS.

\begin{tabular}{|c|c|c|c|c|c|c|c|c|}
\hline & \multicolumn{3}{|c|}{ Flow rate } & \multicolumn{5}{|c|}{$0.65 \mathrm{ml} /$ minutes } \\
\hline \multirow{3}{*}{ HPLC } & \multicolumn{3}{|c|}{ Autosampler injection volume } & \multicolumn{5}{|c|}{$10 \mu 1$} \\
\hline & \multicolumn{3}{|c|}{ Autosampler temperature } & \multicolumn{5}{|c|}{$25^{\circ} \mathrm{C}$} \\
\hline & \multicolumn{3}{|c|}{ Column oven temperature } & \multicolumn{5}{|c|}{$30^{\circ} \mathrm{C}$} \\
\hline \multirow{4}{*}{ Chromatography } & \multicolumn{3}{|c|}{ Mobile phase } & \multicolumn{4}{|c|}{$30 \%$ of $5 \mathrm{mM}$ ammonium chloride : $70 \%$ Methanol } & \\
\hline & \multicolumn{3}{|c|}{ Column type } & \multicolumn{4}{|c|}{ ACE C8 column $(50 \times 4.6 \mathrm{~mm}$ i.d. $5 \mu \mathrm{m})$} & \\
\hline & \multirow{2}{*}{\multicolumn{3}{|c|}{ Expected retention time: }} & \multicolumn{5}{|c|}{ TS: 0.91 minutes } \\
\hline & & & & \multicolumn{5}{|c|}{ HCT (IS): 0.79 minutes } \\
\hline \multirow{3}{*}{ MRM detection } & & & Q1 mass & Q2 mass & Dwell time & DP & $\mathrm{CE}$ & EP \\
\hline & & & 228.842 & 148.6 & 150.0 & -45 & -30 & -15 \\
\hline & & & 295.800 & 268.5 & 150.0 & -45 & -28 & -10 \\
\hline \multirow{2}{*}{ Source/gas } & CUR & CAD & & & & GS1 & & \\
\hline & 25 & 8 & & & & 55 & & \\
\hline
\end{tabular}

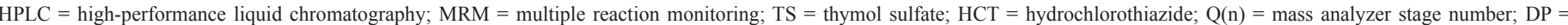

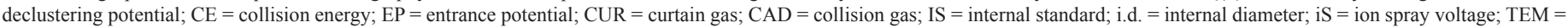
temperature of ion source; GS1 = nebulizing gas; GS2 = drying gas.

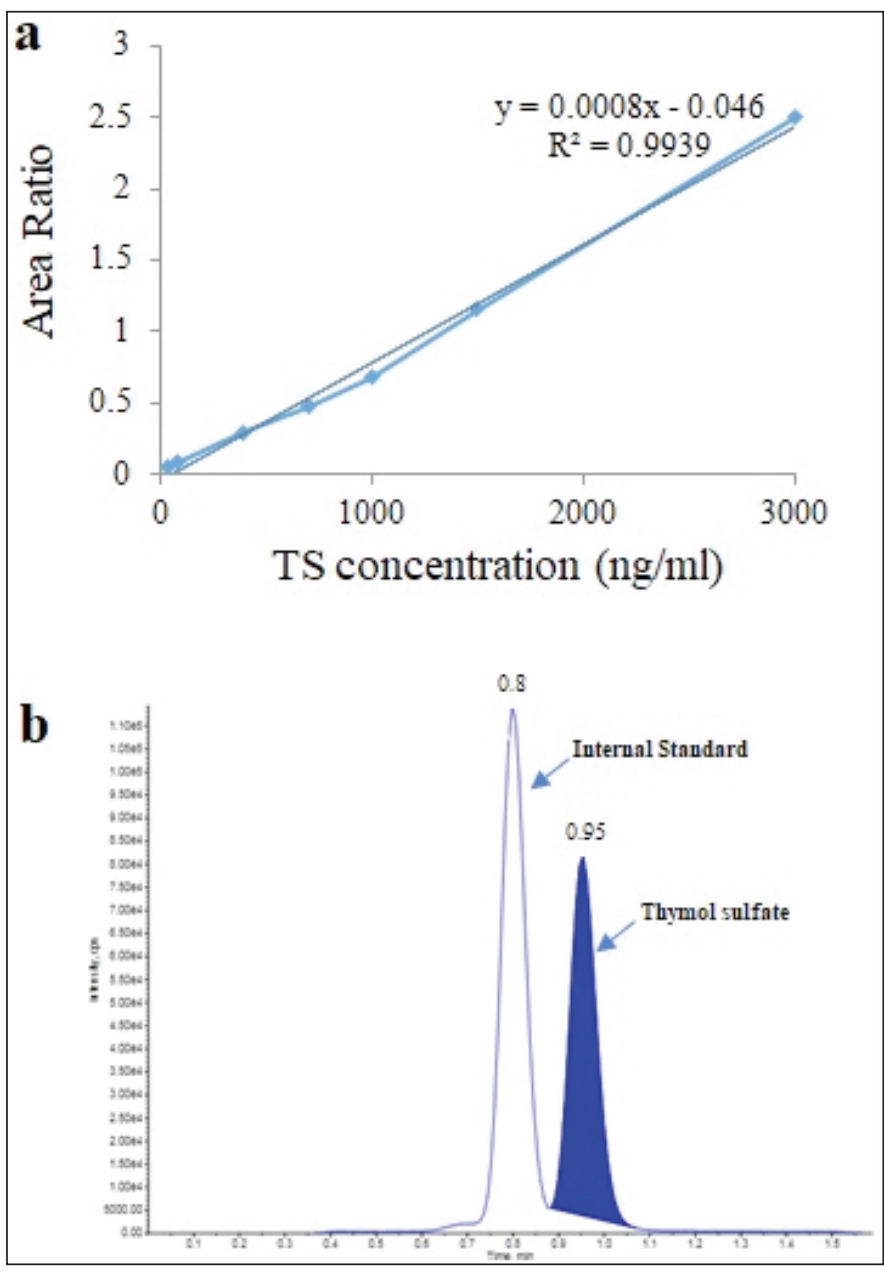

Figure 2. (A) Calibration curve of linearity of intraday validation; (B) LC/MS chromatogram of TS and internal standard. TS $=$ thymol sulfate. at $40^{\circ} \mathrm{C}$ in a water bath under airflow for 30 minutes. The obtained residue was reconstituted with $250 \mu \mathrm{l}$ of $0.5 \mathrm{mM}$ ammonium chloride: $\mathrm{MeOH}$ (30:70) and vortexed for 1 minute to ensure complete dissolution and readiness for analysis.

\section{Validation of TS method of analysis}

The validation was carried out based on the European Medicines Agency (EMA) guidelines (EMA, 2012). The validation assessed included specificity, linearity, intraprecision, and accuracy.

Specificity

The selectivity was evaluated by analyzing 12 spiked matrix samples at the LLOQ level from six different rat plasma sources.

\section{Linearity and LLOQ}

The linearity was measured by assaying calibration curves in rat plasma and was plotted as the peak area ratio (analyte peak area/IS peak area) versus nominal concentrations. Curves were fitted by least-squares linear regression and the acceptable criterion was that correlation coefficient $\left(r^{2}\right)$ was $\geq$ 0.99 . The concentrations of calibration standards defined a lowest concentration as LLOQ compared with nominal concentrations. The accuracy was within $\pm 20 \%$ and the precision was $\leq 20 \%$.

Accuracy and precision

Accuracy and precision evaluations were determined by the analysis of six replicate Quality Control (QC) samples at four levels. The relative error (RE\%) was used to assess accuracy, while the calculation of the coefficient of variation (CV\%) was used to assess precision. The acceptance criteria of intraday precision for LLOQ and three QC levels were less than $15 \%$ and the accuracy was within $\pm 15 \%$ (Table 2 ). 


\section{RESULTS}

\section{Method validation}

The intraday precision and accuracy values of QC and LLOQ samples are presented in Table 2. The intra-assay mean precision was less than $7.01 \%$ and the accuracy range was between 87.32 and $113.70 \%$. The calibration curve of concentration of TS over the range of $40-3,000 \mathrm{ng} / \mathrm{ml}$ was established and linearity was obtained with $r^{2} \geq 0.993$. The typical equation of the TS calibration curve was $y=0.0008 x-0.046$, where $y$ represents the ratio of the peak area of the analyte to that of IS and $x$ represents the plasma concentration (Fig. 1). The LLOQ of TS in rat plasma was $40 \mathrm{ng} / \mathrm{ml}$; the sensitivity was enough to determine the plasma concentration and investigate TS concentration in both rat maternal and fetal tissues. The mean intra-assay precision (CV\%) at LLOQ concentration was $2.31 \%$, with a calculated RE of $12.65 \%$.

\section{In-life observation of dams following $\mathrm{TE}$ and $\mathrm{T}$ administration}

Treatment with $\mathrm{TE}$ and $\mathrm{T}$ in the three selected dose levels was generally well tolerated showing no external signs of toxicity. An increase in relative weights of rats was recorded at gestation day 0 and the termination day was considered clinically nonsignificant for all groups. No mortality cases were recorded in dams in all groups.

\section{Postmortem examination and gross observations of uterine, placenta, and fetuses}

No signs of abortion or premature delivery prior to scheduled caesarean sectioning were noted in dams. Uteri of each dam submitted to macroscopic examination reported no abnormalities. The average relative weights of uteri and placentas

Table 2. Accuracy and precision for intraday QC samples.

\begin{tabular}{lcccc}
\hline \multirow{2}{*}{ Sample name } & \multicolumn{2}{c}{ Concentration (ng/ml) } & \multirow{2}{*}{ RE\% } & \multirow{2}{*}{ CV\% } \\
\cline { 2 - 4 } & Nominal & Calculated & & \\
\hline LLOQ & 40 & 45.06 & 12.65 & 2.31 \\
$\mathrm{QC}_{\text {Low }}$ & 120 & 127.78 & 6.49 & 7.01 \\
$\mathrm{QC}_{\text {Medium }}$ & 1,500 & $1,603.93$ & 6.93 & 3.91 \\
$\mathrm{QC}_{\text {High }}$ & 2,400 & $2,731.81$ & 13.83 & 1.04 \\
\hline SD = standard deviation; & $\mathrm{RE}=$ accuracy (\% mean relative error); CV = precision \\
(\% coefficient of variation). & & &
\end{tabular}

Table 3. Accuracy percentage-based intraday-measured TS concentrations of standard points.

\begin{tabular}{|c|c|c|c|c|c|}
\hline \multicolumn{2}{|c|}{ Concentration (ng/ml) } & \multirow{2}{*}{ TS peak area } & \multirow{2}{*}{ IS peak area } & \multirow{2}{*}{ Area ratio } & \multirow{2}{*}{ RE\% } \\
\hline Nominal & Calculated & & & & \\
\hline 40 & 43.75 & 283,921 & $6,042,777$ & 0.04 & 9.40 \\
\hline 80 & 90.96 & 489,786 & $5,920,521$ & 0.08 & 13.70 \\
\hline 400 & 377.12 & $1,843,609$ & $6,157,355$ & 0.29 & -5.72 \\
\hline 700 & 611.22 & $2,814,388$ & $5,904,200$ & 0.47 & -12.68 \\
\hline 1,000 & 876.40 & $4,177,082$ & $6,165,617$ & 0.67 & -12.36 \\
\hline 1,500 & $1,509.93$ & $6,847,386$ & $5,919,307$ & 1.15 & 0.63 \\
\hline 3,000 & $3,245.26$ & $15,211,495$ & $6,083,993$ & 2.50 & 8.17 \\
\hline
\end{tabular}

TS = thymol sulfate; IS = internal standard; RE = accuracy ( $\%$ mean relative error $)$; $R^{2}>0.993 ;$ slope $=0.0008 ;$ intercept $=-0.046 ;$ correlation $=0.996$. were recorded and no significant difference $(p>0.05)$ was found in the weight of uterus in all treated groups in comparison to control (Table 4). However, induction in placenta masses of TEtreated groups was considered significant ( $p$ value $>0.01$ ), despite neglected changes in placenta weights of T-treated groups, in comparison to corresponding control groups. Uterine content was examined for embryonic/fetal deaths and viable live neonates, recording one dead fetus in a 20x TE-treated mother.

The number and distribution of intrauterine implantations were recorded for each treated group and the resorption index was calculated (Table 4). When compared to corresponding control, implantation sites were found to be increased in T-treated groups, despite the dosing concentration. On the contrary, a low number of implanted fetuses was evident in rats of 20x TE group. The average fetal count in dams of other dosing groups including TE- and T-treated was insignificant when compared to that of the control $(p<0.05)$. Total resorption in fetuses of pregnant rats was found to be the highest in dams treated with 20x TE, specifically, $21.42 \%$ higher when compared to the control group.

Examination of neonates was made for alterations and malformation or anomalies where each neonate was grossly examined for external defects, including the presence or absence of the head, cleft palate, limbs, and tail. The general conditions of neonates were all favorable, and no anomalies were clear to sight. One mummified fetus among the dams administered $20 \mathrm{x}$ TE showed signs of shrinkage (Fig. 3). The average weights of fetuses were significantly increased in all TE-treated groups $(p>$ $0.01)$. On the other hand, among fetuses of dams treated with $1 \mathrm{x}$ $\mathrm{T}$, low birth weights were observed (Table 4). The higher doses of $\mathrm{T}$, however, did not show any significant changes in body weights compared to the control group.

\section{Plasma concentration of TS in rat dams and fetuses}

The plasma concentrations of TS in dams and fetuses for each dosing group are shown in Figure 4. Trend-like proportional increase in TS concentration was observed in maternal and fetal samples. Maternal concentrations were found to be statistically significant between the $1 \mathrm{x}$ TE dose and the other groups $(p<0.01)$. Furthermore, the difference in TS fetal concentration between $1 \mathrm{x}$ TE and T dose levels was found to be significant $(p<0.05)$. A dose-dependent increase in TS concentration in fetal tissue was noted in both treatments, where the difference in TS concentration
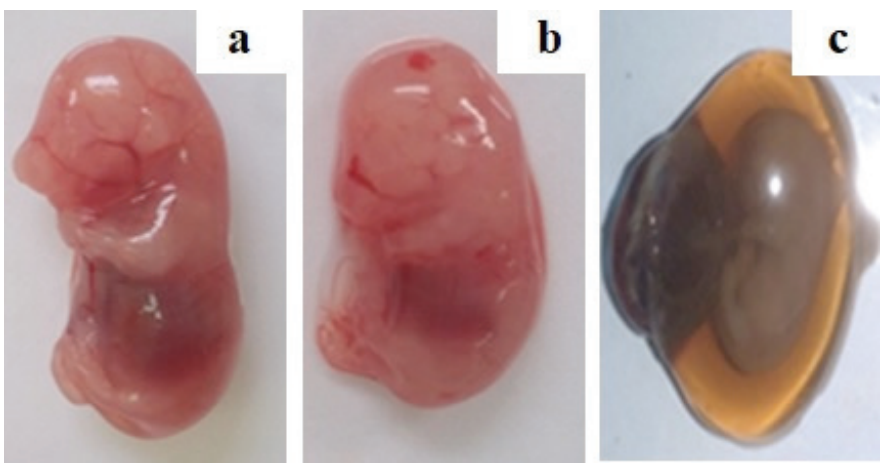

Figure 3. Representative rat maternal fetuses. (a) Control; (b) low birth weight fetus 1x T; (c) mummified rat maternal fetus treated with $20 \mathrm{x}$ TE. TE $=$ thyme extract; $\mathrm{T}=$ thymol compound. 
Tafesh et al. / Journal of Applied Pharmaceutical Science 11 (01); 2021: 144-151

Table 4. Postmortem examination and gross observations of rat uterine, placenta, and fetuses.

\begin{tabular}{|c|c|c|c|c|c|c|c|c|}
\hline & Control (DW) & $1 \times \mathrm{TE}$ & 10x TE & 20x TE & Control (PG) & $1 \times T$ & $10 \times T$ & $20 \times T$ \\
\hline Relative uterus weight (g) & $0.043 \pm 0.003$ & $0.051 \pm 0.008$ & $0.047 \pm 0.004$ & $0.036 \pm 0.01$ & $0.030 \pm 0.007$ & $0.030 \pm 0.01$ & $0.030 \pm 0.01$ & $0.031 \pm 0.01$ \\
\hline Relative placenta weight (g) & $0.38 \pm 0.06$ & $0.49 \pm 0.01^{* *}$ & $0.49 \pm 0.04^{* *}$ & $0.57 \pm 0.03^{* * *}$ & $0.43 \pm 0.06$ & $0.39 \pm 0.05$ & $0.52 \pm 0.2$ & $0.42 \pm 0.12$ \\
\hline Total early resorbed sites & 1 & 1 & 1 & 6 & 0 & 1 & 1 & 2 \\
\hline Total late resorbed sites & 0 & 0 & 0 & 1 & 0 & 0 & 1 & 0 \\
\hline Mean live neonates & $8.6 \pm 2.33$ & $9.0 \pm 2.54$ & $9.4 \pm 1.14$ & $4.2 \pm 2.5^{*}$ & $6.5 \pm 1.5$ & $7.0 \pm 4.06$ & $6.14 \pm 3.71$ & $6.33 \pm 3.39$ \\
\hline Neonatal weight (g) & $1.37 \pm 0.33$ & $1.99 \pm 0.07^{* * *}$ & $1.85 \pm 0.18^{* *}$ & $1.92 \pm 0.12^{* *}$ & $1.26 \pm 0.06$ & $0.74 \pm 0.02^{*}$ & $1.24 \pm 0.15$ & $1.05 \pm 0.36$ \\
\hline
\end{tabular}

$\mathrm{DW}=$ distilled water; $\mathrm{TE}=$ thyme extract; $\mathrm{PG}=$ polyethylene glycol; $\mathrm{T}=$ thymol compound.

${ }^{*} p$ value $>0.05 ; * * p$ value $>0.01 ; * * * p$ value $>0.001$ using $t$-test, in comparison to corresponding control using $t$-test $(n=5)$.

between the low and the high doses was reported to be significant $(p<0.05$; Fig. 4).

\section{DISCUSSION}

The current study aimed to investigate the safety of ingesting standardized thyme extract during pregnancy through studying it in rats. The influence of TS, a metabolite of thyme, was evident in the fetal fraction which indicates the capacity of TS of crossing the placental barrier. Such an event reflected potential toxicity to the pregnancy and the fetuses accumulated by the incautious use of medical plants. Owing to their therapeutic effects, many medicinal plants are used by pregnant women and are often consumed based on traditional habits or personal experience, and in most cases, information considering the safety of plant use during pregnancy is questioned (Adams et al., 2010; Illamola et al., 2019). In addition, herbal medicines are available over the counter, making them easily accessible and putting pregnant women under risk when self-prescribed. Thyme herb, namely T. vulgaris L. or thymol-containing herbs such as Origanum syriacum L., are commonly advocated against colds, gastralgia, and anxiety (Illamola et al., 2019). However, the effect of thymolcontaining herbs on the developing fetus is still unknown. Given that the American Herbal Products Association (American Herbal Products Association, 1992) states that thyme is contradicted in pregnancy and previous studies have contradictory reports on its safety (Cohen, 2013; Jyoti et al., 2018; Laelago, 2019), studies on the developmental toxicology of thyme are warranted.

The current study developed and validated an $\mathrm{LC} /$ MS method for detection and quantification of rat plasma TS, a metabolite of T. vulgaris L. extract in fetus and maternal uterine tissues through the placental transmission. This method was found to be robust and reproducible and had a high sensitivity for TS with an LLOQ of $40 \mathrm{ng} / \mathrm{ml}$. Therefore, the plasma was validated over a concentration range of $120-2,400 \mathrm{ng} / \mathrm{ml}$ and good linearity was obtained with $r^{2}>0.993$. The mean intra-assay precision $(\mathrm{CV} \%)$ was less than $7.01 \%$ with mean values of accuracy between $-12.68 \%$ and $13.7 \%$, which revealed that the current method was reliable, accurate, and reproducible. The validated LC/MS method was used to determine quantities of thyme metabolite, namely TS in rat maternal and fetal effluent samples, and provided sufficient and consistent readings. To our knowledge, this is the first study to determine TS in rat plasma through LC/MS and report insights into the transplacental transfer of TS.

This study has demonstrated that exposure to thymolcontaining compounds, represented in the study by $\mathrm{TE}$ or $\mathrm{T}$ administration at concentrations that are equivalent to those
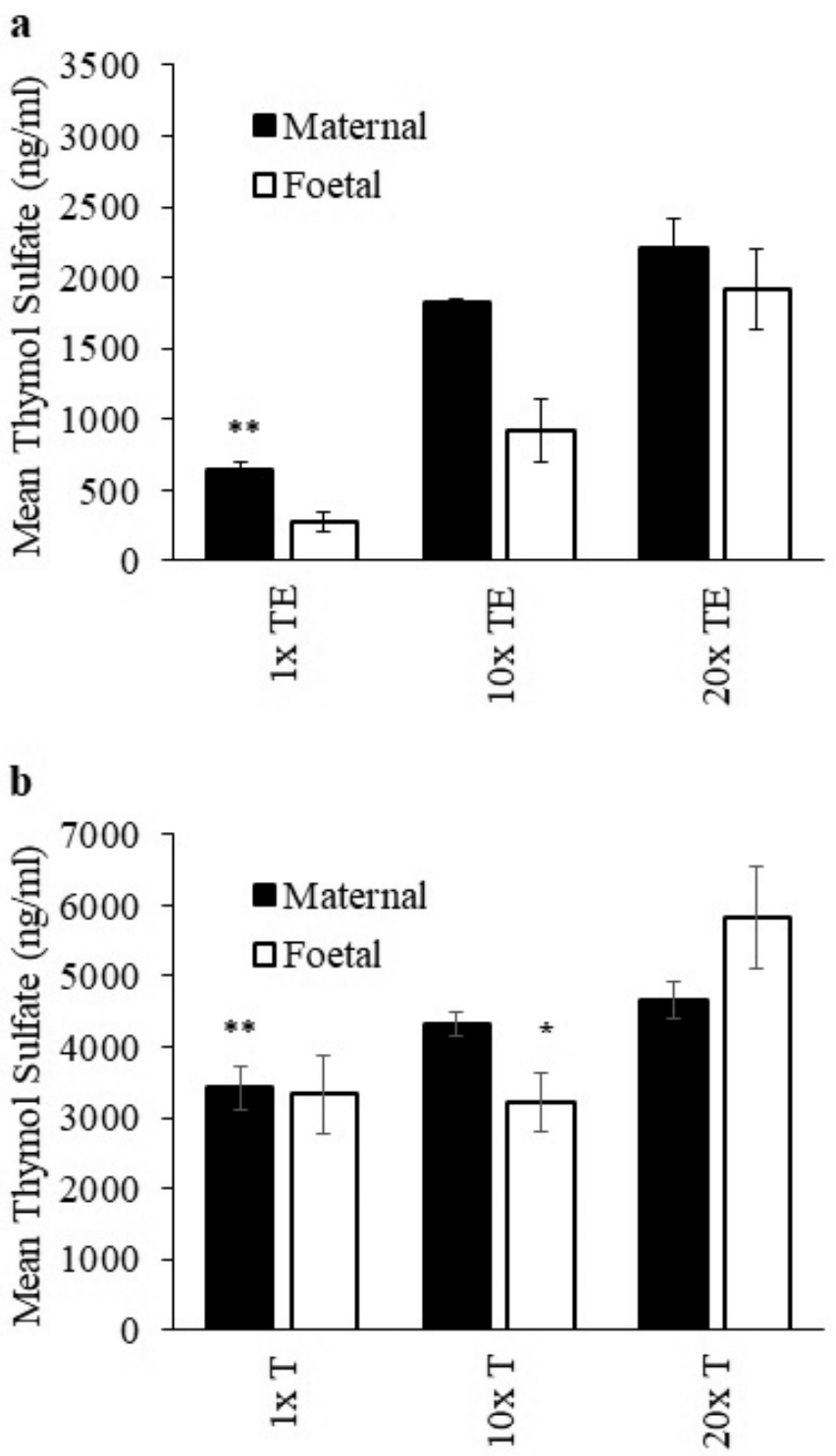

Figure 4. Mean TS concentration in maternal and fetal effluent extracts treated with three dose levels of (a) TE and (b) T. ** = significant in comparison to maternal 20x $(p<0.01) ; *=$ significant in comparison to fetal 20x T $(p<0.05)$. $\mathrm{TE}=$ thyme extract; $\mathrm{T}=$ thymol compound.

advocated for the treatment of cough or bronchitis, could result in several changes to fetal development. However, clinicians usually prescribe thyme products to pregnant women at a dose equivalent 
to $93.75 \mathrm{mg} / \mathrm{kg} /$ day (based on $15 \mathrm{~g} / 100 \mathrm{ml}$ syrups). Therefore, in the current investigation, the dose of $93.75 \mathrm{mg} / \mathrm{kg} / \mathrm{day}$ of TE was considered as the RDD. Thymol has been reported to be metabolized by CYP450 isoform 2A6 (Dong et al., 2012) and then sulfated by sulfotransferase producing TS, which was reported as the predominant metabolite of thymol in plasma (Kohlert et al., 2002). Thus, TS was selected as a quality marker of TE and T.

Nonetheless, by observing potential adverse effect levels, no alterations on maternal well-being were found nor to weight gain during the dosing period. Although the mean change in placenta weights was not evident in groups treated with thymol, relative placenta weights in thyme extract-treated dams were significantly higher when compared to the corresponding control. It has been previously reported that thyme extract acts as an appetite stimulant; thus, heavier placenta weight could be related to enhanced maternal nutrition accounted to the enhanced appetite after treatment with thyme extract (Dauqan and Abdullah, 2017). This could have impacted placental growth, thereby affecting uteroplacental blood flow and nutrient uptakes by the fetus, all of which play an important role in fetal growth, metabolism, and birth weight (Redmer et al., 2004). However, there was no apparent concentration-related effect as both compounds showed similar relative uterus weights.

The mean number of implantations and litter size per dam did not differ among all groups except for 20x TE group, where the mean live neonate number was reduced to more than a half. In addition, the number of visible early resorptions in 20x TE group was six times higher than the lower doses. The embryonic observations reported no phenotypic anomalies on fetal development among most fetuses. However, the administration of $1,875 \mathrm{mg} / \mathrm{kg}(20 \mathrm{x}) \mathrm{TE}$ caused a higher rate of resorption which reached $21.42 \%$ and a lower number of fetuses, in addition to one case of intrauterine death found as the early mummified fetus. Such observations were parallel to high TS quantities in 20x TE fetuses in comparison to TS concentrations that reached fetuses in rats treated with lower TE doses. On the other hand, resorption in T-treated groups was twice higher in doses higher than $1 \mathrm{x}$ T. Furthermore, low birth weight was evident in fetuses of dams receiving RDD of T. However, growth retardation was evident in pups of $1 \mathrm{x}$ T-treated group. It has been suggested that the embryotoxicity represented as resorption may be a result of insufficient hormone concentration and deficient placenta function or may be due to the direct effect of the drug on the embryos (El Gendy et al., 2015). Furthermore, levels of the thyme metabolite TS were considerably high in fetuses of T-treated rats, suggestive of the higher capacity of thymol in crossing the placenta and reaching the fetal effluent. These data combined with the finding of one mummified fetus within the 20x TE group need further indepth investigation to confirm the raised potential risk of thyme and thymol extract on pregnancy progression. Such an incident needs further investigation in order to inspect the impact of thymol on prenatal development. However, manifestations could be linked to findings of a dose-dependent increase in TS formation in maternal rat plasma. The proportional increase in the transplacental transfer of TS was observed by the increase in TE concentration. As for groups treated with $\mathrm{T}$, concentrations of the metabolite were significantly higher in fetuses of $20 \mathrm{x} T$ in comparison to fetuses of lower concentrations.
Presence of TS in fetal tissues reflects its ability to cross the placenta barrier due to its physicochemical properties. Molecules that are relatively lipophilic with low-protein binding and low degree of ionization and have Molecular weight (MW) of less than 600 Da permeate, as is the case with TS, readily cross the placenta layers (Ala-Kokko et al., 1993).

As the fetal kidney develops, more drug metabolites are excreted into the amniotic fluid, leading to the accumulation of drugs within the fetus which is called the ion trapping phenomenon, where more acidic nature of fetal plasma $\mathrm{pH}$ is evident compared to maternal plasma. In addition, weak bases and nonionized (lipophilic) molecules can diffuse across the placental barrier and become ionized in the more acidic fetal blood, leading to a net movement from the maternal to fetal systems (Anger et al., 2011).

When thyme-containing remedy is prescribed or consumed, as in most herbal extracts, one should be conscious that constituents other than the active medicinal compound could be present in the extract and could be harmful or toxic. The major phytochemical constituents found in TE include essential oils such as thymol, carvacrol, and p-cymene (Dauqan and Abdullah, 2017) and flavonoids including apigenin and luteolin (Javed et al., 2013; Stahl-Biskup and Venskutonis, 2004), in addition to phenolic acids such as rosmarinic acid (Zgórka and Glowniak, 2001). Several studies have investigated such components which can also be found in other plants. In preclinical studies, phenols, for example, were reported to be embryotoxic and fetotoxic, but not teratogenic. Furthermore, phenols generally do not cause developmental defects except at doses that cause maternal toxicity; as for terpenes, they can be chemically classified as alcohols, hydrocarbons, ketones, and epoxides with low cytotoxicity (Mendanha et al., 2013).

\section{CONCLUSION}

A selective LC/MS method was successfully developed and validated for quantitative analysis of TS in rat plasma and fetal tissue with high sensitivity and suitable reported LLOQ for TS that reached $40 \mathrm{ng} / \mathrm{ml}$. The current investigation revealed that high doses of thyme extract and its chronic use may alter the placental functions and therefore could affect the growth of fetuses. Based on the results of this study, pregnant females can be advised clinically to use thyme extract only within the recommended doses. Preclinically, further research is still warranted to evaluate the safety of thymol and other thyme constituents on modulating the placental barrier and protein binding. A detailed histological investigation of thyme effects on placental and neonatal vital organs such as the liver and kidneys is also recommended.

\section{ACKNOWLEDGMENTS}

The authors would like to thank the University of Petra for funding this project and providing research assistance, staff of the Jordan Center for Pharmaceutical Research (JCPR) and the animal house staff at the Applied Science University for their valuable assistance.

\section{CONFLICT OF INTEREST}

The author declared no potential conflicts of interest with respect to the research, authorship, and/or publication of this article. 


\section{FUNDING}

This project was supported by the Deanship of Scientific Research, University of Petra, Amman, Jordan (Grants nos. 4-42019, 2-4-2019, and 2-4-2018).

\section{AUTHOR CONTRIBUTIONS}

All authors made substantial contributions to conception and design, acquisition of data, or analysis and interpretation of data; took part in drafting the article or revising it critically for important intellectual content; agreed to submit to the current journal; gave final approval of the version to be published; and agree to be accountable for all aspects of the work.

\section{ETHICAL APPROVAL}

This study is approved by the ethical committee of the University of Petra, Amman, Jordan (ethical approval number: 2016/2017-66).

\section{REFERENCES}

Adams J, Lui CW, Sibbritt D, Broom A, Wardle J, Homer C, Beck S. Women's use of complementary and alternative medicine during pregnancy. Obstet Anesth Dig, 2010; 30(4):238.

Ala-Kokko T, Våhåkangas K, Pelkonen O. Placental function and principles of drug transfer. Acta Anaesthesiol Scand, 1993; 37:047-9.

American Herbal Products Association. AHPA: herbs of commerce. American Herbal Products Association, Silver Spring, MD, p $552,1992$.

Anger GJ, Costantine MM, Piquette-Miller M. Pharmacokinetics in pregnancy. In: Gupta RC (ed.). Reproductive and developmental toxicology, Academic Press, Cambridge, MA, pp 039-45, 2011.

Basch E, Ulbricht C, Hammerness P, Bevins A, Sollars D. Review of thyme (Thymus vulgaris). J Herb Pharmacother, 2006; 4(1):049-67.

Basch E, Ulbricht C, Hammerness P, Bevins A, Sollars D. Thyme (Thymus vulgaris L.), thymol. J Herb Pharmacother, 2004; 4:049-67.

Cohen PA. American medical association. Arch Intern Med, 2013; 172(13):2012-3.

Dauqan EMA, Abdullah A. Medicinal and functional values of thyme (Thymus vulgaris L.) Herb. J Appl Biol Biotechnol, 2017; 5(02):017-22. https://www.ema.europa.eu/en/documents/scientificguideline/guideline-bioanalytical-method-validation_en.pdf

Dong RH, Fang ZZE, Zhu LL, Ge GB, Cao YF, Li XB, Hu CM, Yang L, Liu ZY. Identification of CYP isoforms involved in the metabolism of thymol and carvacrol in human liver microsomes (HLMs). Pharmazie, 2012; 67(12):1002-6.

El Gendy MM, Kandil AM, Helal MA, Zahou FM. The teratogenic effects of imatinib mesylate on rat fetuses. Toxicol Rep, 2015; 2:654-63.

Gavliakova S, Biringerova Z, Buday T, Brozmanova M, Calkovsky V, Poliacek I, Plevkova J. Antitussive effects of nasal thymol challenges in healthy volunteers. Respir Physiol Neurobiol, 2013; 187: $104-7$.

Gray MJ, Chang D, Zhang Y, Liu J, Bensoussan A. Development of liquid chromatography/mass spectrometry methods for the quantitative analysis of herbal medicine in biological fluids: a review. Biomed Chromatogr, 2010; 24:091-103.

Hosseinzadeh S, Jafarikukhdan A, Hosseini A, Armand R. The application of medicinal plants in traditional and modern medicine: a review of Thymus vulgaris. Int J Clin Med, 2015; 06(09):635-42.

Illamola SM, Amaeze OU, Krepkova LV, Birnbaum AK, Karanam A, Job KM, Bortnikova VV, Sherwin CMT, Enioutina EY. Use of herbal medicine by pregnant women: what physicians need to know. Front Pharmacol, 2019; 10(1):001-16.

Javed H, Erum S, Tabassum S, Ameen F. An overview on medicinal importance of Thymus vulgaris. J Asian Sci Res, 2013; 3(10):974-82.
Jyoti, Dheer D, Singh D, Kumar G, Karnatak M, Chandra S, Prakash Verma V, Shankar R. Thymol chemistry: a medicinal toolbox. Curr Bioact Compd, 2018; 15(5):454-74.

Kohlert C, Schindler G, März RW, Abel G, Brinkhaus B, Derendorf H, Gräfe EU, Veit M. Systemic availability and pharmacokinetics of thymol in humans. J Clin Pharmacol, 2002; 42(7):731-7.

Laelago T. Herbal medicine use during pregnancy: benefits and untoward effects. In: Builders P (ed.). Herbal medicine, IntechOpen Limited, London, UK, 2019

Leonardo E, Matos S, Rubio N, Pereira C, Oliveira PR De, Daemon E. Histopathological changes in the liver and thyroid of mice (Mus musculus) caused by the acaricides: fipronil and thymol. J Histol Histopathol, 2017; 4(1):9.

Louik C, Gardiner P, Kelley K, Mitchell AA. Use of herbal treatments in pregnancy. Am J Obstet Gynecol, 2010; 202(5):439.e1-10.

Marston A, Hostettmann K. Natural product analysis over the last decades. Planta Med, 2009; 75:672-82.

Mendanha SA, Moura SS, Anjos JLV, Valadares MC, Alonso A. Toxicity of terpenes on fibroblast cells compared to their hemolytic potential and increase in erythrocyte membrane fluidity. Toxicol In Vitro, 2013; 27(1):323-9.

OECD/OCDE. Guideline no 414 - Prenatal developmental toxicity study, OECD Guidelines for the Testing of Chemicals. OECD, Paris, France, pp 001-14, 2018.

Redmer DA, Wallace JM, Reynolds LP. Effect of nutrient intake during pregnancy on fetal and placental growth and vascular development. In: Cardoso R, Donadeu X. Domestic animal endocrinology, 2004; 199 217

Sharma V, Sarkar IN. Bioinformatics opportunities for identification and study of medicinal plants. Brief Bioinform, 2013; 14(2):238-50

Stahl-Biskup E, Venskutonis RP. Thyme. In: Peter KV (ed). Handbook of herbs and spices, CRC Press, Cambridge, England. pp $297-$ $321,2004$.

Verrett MJ, Scott WF, Reynaldo EF, Alterman EK, Thomas CA. Toxicity and teratogenicity of food additive chemicals in the developing chicken embryo. Top Catal, 1980; 56(2):265-73.

Wang H, Yang LL, Hu YF, Wang BW, Huang YY, Zhang C, Chen $\mathrm{YH}, \mathrm{Xu}$ DX. Maternal LPS exposure during pregnancy impairs testicular development, steroidogenesis and spermatogenesis in male offspring. PLoS One, 2014; 9(9):e106786.

Woo CSJ, Lau JSH, El-Nezami H. Herbal medicine: toxicity and recent trends in assessing their potential toxic effects. Adv Bor Res, 2012; $62: 365-84$.

World Health Organization. WHO monographs on selected medicinal plants. Essent Med Heal Prod Inf Portal, 2nd edition, Vol 1, World Health Organization, Geneva, Switzerland, pp 183-94, 1999.

Wu H, Guo J, Chen S, Liu X, Zhou Y, Zhang X, Xu X. Recent developments in qualitative and quantitative analysis of phytochemical constituents and their metabolites using liquid chromatography-mass spectrometry. J Pharm Biomed Anal, 2013; 72:267-91.

Zgórka G, Glowniak K. Variation of free phenolic acids in medicinal plants belonging to the Lamiaceae family. J Pharm Biomed Anal, 2001; 26(1):079-87.

How to cite this article:

Tafesh ZQ, Mansoor KA, Qinna NA, El-Hajji FD, Arafat TA, Abu-Qatouseh LF, Ghanim BY, Bashiti IA, Mallah EM. Reproductive safety assessment of Thymus vulgaris L. extract and quantification of thymol sulfate in pregnant rats and fetuses using a validated LC/MS method of analysis. J Appl Pharm Sci, 2021; 11(01):144-151. 